\title{
Evidence-Based Psychodynamic Therapies for the Treatment of Patients With Borderline Personality Disorder
}

Svenja Taubner ${ }^{\mathrm{a}}$, Jana Volkert ${ }^{\mathrm{a}}$

[a] University Hospital Heidelberg, University of Heidelberg, Heidelberg, Germany.

Clinical Psychology in Europe, 2019, Vol. 1(2), Article e30639, https://doi.org/10.32872/cpe.v1i2.30639

Received: 2018-10-17 • Accepted: 2019-05-24 • Published (VoR): 2019-06-28

Handling Editor: Cornelia Weise, Philipps-University of Marburg, Marburg, Germany

Corresponding Author: Svenja Taubner, University Hospital Heidelberg, University of Heidelberg, Institute for Psychosocial Prevention, Bergheimer Str. 54, 69115 Heidelberg, Germany. Tel.: 0049(0)6221-56-4700. E-mail: svenja.taubner@med.uni-heidelberg.de

\begin{abstract}
Background: Borderline Personality Disorder (BPD) is a serious health issue associated with a high burden for the individual and society. Among the "Big Four" of evidence-based treatments for patients with BPD are two psychodynamic therapies that have evolved from classic psychoanalytic treatment with a change of setting and change of focus: Transference-Focused Psychotherapy (TFP) and Mentalization-Based Treatment (MBT).

Aims: This overview provides a comparison of the two treatments in terms of stance, clinical concepts, costs and key interventions. Furthermore, the current literature on the efficacy of both treatments is reviewed.

Results: While TFP focuses on the content of disintegrated representations of self and other, MBT focuses on the processing of mental states. Both treatments diverge in their clinical concepts and interventions for the treatment of BPD.

Conclusion: Although both treatments are regarded as effective in treating BPD, no direct comparison of both treatments has been made so far. Future studies are needed to investigate mechanisms of change and derive recommendations for a differential indication.
\end{abstract}

\section{Keywords}

psychodynamic psychotherapy, borderline personality disorder, mentalization-based treatment, transferencefocused psychotherapy, efficacy, clinical concepts 


\section{Highlights}

- Specialized therapies for BPD have favorable drop-out rates and outcome compared to non-specialized ones.

- MBT and TFP have very diverse clinical concepts and interventions for the treatment of BPD.

- Both, MBT and TFP show efficacy in RCTs.

- No trial has directly compared MBT and TFP; there is no evidence base for differential indication.

The Cochrane review (Stoffers et al., 2012) on psychological therapies for Borderline Personality Disorder (BPD) lists several approaches as 'probably effective' in treating BPD. Four psychological treatments are described as evidence-based, the "Big Four". Among those two psychodynamic treatments are listed: Mentalization-Based Therapy (MBT) and Transference-Focused Psychotherapy (TFP). Both represent the trend in psychodynamic therapies to develop disorder-specific treatments that can be tested for efficacy in contrast to a classic, more transdiagnostic approach. Furthermore, psychodynamic therapies have been developed that deviate from the classic Freudian conceptualizations of addressing unconscious conflict to improving personality functioning instead. In this paper, we will outline these current developments of psychodynamic treatments among the "Big Four" for BPD as the most prevalent disorder in clinical settings (Torgersen, 2005), due to a lack of trials for other psychodynamic approaches (e.g. Dynamic Deconstructive Psychotherapy or Psychoanalytic Interactional Method). First, we will summarize the common ground of psychodynamic therapies and, secondly, describe the clinical and change theory as well as therapeutic stance and key interventions of the two treatments. In a third step, TFP and MBT will be compared and contrasted with regard to their similarities and differences. The paper concludes with a summary of the current research findings on the efficacy of MBT and TFP for BPD and points out future directions for clinical research of these two approaches. Differences in efficacy to classic psychodynamic treatment will be discussed.

\section{Common Features of Psychodynamic Psychotherapy}

The term psychodynamic psychotherapy was established to describe therapies following the core psychoanalytic principles but with a lower weekly session rate and using a faceto-face setting instead of the classic Freudian couch setting (Whitehorn, Braceland, Lippard, \& Malamud, 1953). Furthermore, psychodynamic psychotherapies establish a treatment focus and limit treatment goals also with regard to symptomatic changes. Par- 
allel to the development of psychodynamic psychotherapy, clinical theories were broadened from seeing symptoms not only as a manifestation of unconscious conflicts but also as impairments in personality functioning and disturbed relationships (OPD-2, OPD Taskforce, 2008). Following the demands of evidence-based medicine, disorder-specific treatment manuals were established, e.g. for the treatment of panic disorders (Milrod et al., 2007) and depression (Lemma, Target, \& Fonagy, 2011). The core ideas of psychodynamic therapies remained the following (Shedler, 2010):

1. Focus on emotions and affect

2. Exploration of aspects that patients tend to avoid (e.g. painful and threatening aspects of experience), which is called defense or resistance in psychoanalytic terms

3. Identification of recurrent topics or themes with regard to self, other, relationships, etc.

4. Discussion of past experiences that help to contextualize current experiences

5. Focus on relationships, especially the therapeutic relationship

6. Exploration of dreams, phantasies and wishes

All of these aspects can also be found in MBT and TFP; however, there is a shift of focus in the treatment of BPD to emphasize the "here-and-now" instead of discussing past events. Both treatments work very explicitly with the current therapeutic relationship and the exploration of dreams, phantasies and wishes is not central for the therapeutic process, at least at the beginning of treatment. Furthermore, psychodynamic therapies follow the goal to change distorted representations of self, other or relationships in a quite comparable way to Cognitive-Behavioral Therapy (CBT). These can be distinguished at the level of intervention: while CBT aims to change patients' dysfunctional beliefs at a micro level, psychodynamic therapies reach out to change personality aspects e.g. with regard to depression at a macro level (Luyten, Blatt, \& Fonagy, 2013). Both aim to change the content of representations. However, because a large number of patients do not correspond to a treatment approach focusing on the content of representations, recently in both therapeutic schools, new therapies have been developed that shift from addressing content to the processing of mental states itself (e.g. how we think and interpret instead of what we think). In this paper, we will regard TFP as a primarily content-focused treatment, whereas MBT focuses more on the processing of mental states, which can be regarded in line with the third wave therapies in CBT. Before comparing these two specific treatment approaches- TFP and MBT-, we will summarize the core symptoms and burden of BPD. 


\section{Borderline Personality Disorder}

$\mathrm{BPD}$ is a severe health issue characterized by at least five of the following nine criteria (Diagnostic and Statistical Manual of Mental Disorders [5th ed.; DSM-5]; American Psychiatric Association, 2013): a) unstable relationships, b) inappropriate anger, c) frantic effort to avoid abandonment, d) affective instability, e) impulsivity, f) self-harm/suicidality, g) dysphoria, h) stress-related paranoid thoughts and i) identity disturbance and dissociation. Point prevalence in community samples ranges from $0.7-3.9 \%$ (Trull, Jahng, Tomko, Wood, \& Sher, 2010), lifetime prevalence is around 6\% (Grant et al., 2008). In a recent meta-analysis with $n=66,914$ included individuals from community samples of 9 studies in Western countries the prevalence rate was $1.90 \%$ (Volkert, Gablonski, \& Rabung, 2018). Furthermore, BPD is the most common personality disorder in clinical populations, with prevalence rates of around $10 \%$ in outpatient and $15-25 \%$ in inpatient settings (Torgersen, 2005). BPD is often associated with both comorbid axis I and II disorders: approx. $85 \%$ of BPD patients have a 12-months diagnosis of at least one axis I and $74 \%$ for another axis II disorder (Grant et al., 2008). 69-80\% of BPD patients engage in suicidal behavior and 3-10\% commit suicide with a 50 fold heightened risk in comparison to the general population (Gunderson, Weinberg, \& Choi-Kain, 2013; Leichsenring, Leibing, Kruse, New, \& Leweke, 2011; Oldham, 2006). BPD accounts for $2.2 \%$ of all disability adjusted life years (DALYs), $1.2 \%$ of all DALYs (ranking $3^{\text {rd }}$ in mental disorders in women, and $4^{\text {th }}$ in men) and suicide accounts for $1.0-2.8 \%$ of all DALYs (Victorian Government Department of Human Services, 2005). The burden of BPD on society in terms of productivity losses and other indirect costs is assumed to reach $76.3 \%$ of the total costs (Olesen, Gustavsson, Svensson, Wittchen, \& Jönsson, 2012). Similarly, direct costs of BDP are considered to be higher than in depression or diabetes (Wagner et al., 2013), with average per capita costs ranging between $11,000 €$ and 14,000€ (Salvador-Carulla et al., 2014) per year. In sum, BPD is a severe treatment condition that comes with a high burden for the individual and society. However, treatment of BPD patients is emotionally challenging for therapists, and therapists often decline treatment with this group of patients; although $50 \%$ of therapists agree that a BPD-specific treatment qualification is useful, only $3 \%$ have such a qualification (Jobst, Hörz, Birkhofer, Martius, \& Rentrop, 2010). Thus, there is a high need of training therapists in BPD-specific treatment approaches. The two approaches that will be presented here, MBT (Bateman \& Fonagy, 2016) and TFP (Yeomans, Clarkin, \& Kernberg, 2015), offer an additional training on top of a psychodynamic (or other) psychotherapeutic training that was created to treat BPD more effectively. 


\section{A Content-Focused Psychodynamic Treatment - Transference Focused Psychotherapy}

TFP was developed by Frank Yeomans, John Clarkin, and Otto Kernberg (Yeomans et al., 2015) and is associated with a new conceptual idea of identity formation and personality organization. The aim of treatment is to decrease the symptomatic burden and interpersonal problems in patients with BPD by changing patients' mental representations of others and self that underlies their behavior (Clarkin, Cain, \& Lenzenweger, 2018), to meaningfully improve functioning in the domains of work, studies and profession, and intimate relations (Yeomans et al., 2015).

\section{Clinical Concept}

Personality organization is described as comprising three aspects of personality functioning: identity integration, level of defense mechanisms and degree of reality testing. Following Kernberg (1967), Borderline personality organization is marked by identity diffusion, low level of defenses but mainly intact reality testing. Thereby, identity diffusion is considered central to the clinical understanding of BPD in TFP and is related to a lack of coherence in the individual's experience and understanding of both self and others. Furthermore, social signals are consistently misunderstood because the inner experience of a BPD patient is dominated by aggressive internalized object relations that are split from idealized ones. Thus, identity diffusion is associated with defensive strategies involving dissociation of conscious aspects of conflicting experiences (splitting). The lack of an integrated self is also seen as leading to internal distress and emptiness that lead to patients' attempts to relieve distress through impulsive acting out (Kernberg, 1967). Kernberg's etiology follows the idea of object relation theory that early experiences of self and others are organized by splitting, meaning that positive and negative representations of self and other need to be gradually integrated to achieve normal functioning. In BPD, positive and negative representations of self and other remain separated/ disintegrated because negative representations contain traumatic affects that would possibly destroy positive representations.

\section{Change Theory}

The authors propose that TFP helps patients to establish an increased affect regulation achieved through the growing ability of the patient to psychologically reflect and integrate thoughts, emotions and behavior and to establish positive relationships with others (Kernberg, 2016). This is achieved through a modification of personality structure by linking the dissociated parts of positive and negative representations that are enacted in the therapeutic relationship. The patient's partial representations are experienced in the therapeutic relationship that mirror splitting in the patient (transference). This can lead to a rapid change in the therapeutic relationship, e.g. an idealization of the therapist fol- 
lowed by a fear of being dominated or threatened, which is outside of the patient's awareness. The therapist describes these different states of the relationship and links this with the inner experience of the patient. This way an integration of the split off idealized and persecutory segments of experience can take place, i.e. identity diffusion can be resolved. By addressing the different split-off representations of self and other the therapist engages the patient in thinking and reflecting about their emotional responses and behavior and links this, moment by moment, to the experiences in the therapeutic relationship. This leads to a reflection in the here and now with another person and a growing awareness of how the perception of others is distorted by expectations derived from internal representations. Within this therapeutic process the patient's view of current interpersonal realities becomes more accurate.

\section{Setting}

TFP begins with a verbal contract that serves as a framework to discuss risks to a patient's life (suicidality, self-harm, drug abuse) as well as behavior that potentially limits or hinders the continuation of therapy (leaving a job, insurance, moving to another city). Furthermore, the contract aims at reducing any gains that the patient would take from their symptoms with regard to negative reinforcements. After having agreed on a common contract, two individual sessions weekly are carried out with weekly supervision. The average treatment duration is between two and three years.

\section{Stance}

The therapist takes a more active stance in comparison to classic psychoanalytic treatment by paying more attention to the external reality of the patient (e.g. breaking the contract, antisocial behavior) and selects priority themes that need to be addressed in every session in the material the patient is presenting. The stance is characterized by the "technical neutrality", focusing on the "here-and-now" as well as balancing between exploring and confronting the patient with incompatible views on the one hand and regulation of arousal on the other hand. Technical neutrality describes the general stance of continuously keeping the goal of therapy in mind with an attitude of objective inquiry, to clarify issues without being judgmental. Contradictions in the patient's perception or representations of self and others are observed at three levels: what the patient is saying, how the patient is acting (inside and outside session) as well as the counter-reactions and feelings of the therapist. The latter requires constant monitoring of what belongs to the patient, the therapist and/or their interaction.

\section{Key Interventions}

While the patient is asked to freely associate and disclose any idea that comes to mind, the therapist listens carefully and uses the three following interventions: clarification, 
confrontation and interpretation. Clarification means to thoroughly explore the patient's subjective experience with a special focus on contradictions or conflicts as well as affects in his/her perception of self and others. This intervention aims to promote mentalization of internal states (Yeomans, Levy, \& Caligor, 2013). Confrontations take the therapeutic work to the second level of actively pointing out discrepancies between the three channels of communication (verbal, non-verbal and counter-reactions of the therapist) (Zerbo, Cohen, Bielska, \& Caligor, 2013). Finally, interpretations aim to integrate contradictions by offering a hypothesis for a deeper understanding of the different self and other representations that dominate the patient's thinking and feeling in relationships. In the beginning and middle parts of the treatment, TFP recommends to avoid so-called genetic interpretations that link childhood experiences to current states of mind but stay in the hereand-now.

\section{Time and Costs}

Training comprises 34 weekly seminars over a duration of one year including supervision. This is followed by 6 months' home study and supervision. The cost of the training adds up to 3,000€ (TFP Institute Munich, Germany). Treatment costs for an individual patient may vary from country to country. Number, duration and frequency of therapeutic sessions may approximately range from a minimum of 180 hours (two weekly sessions for one year) to a maximum of 405 sessions (three sessions weekly for three years) based on data from trial therapies. A cost effectiveness study revealed average costs for TFP at about $46.000 €$ and concluded that SF was more cost-effective in comparison to TFP (van Asselt et al., 2008). However, there is no time-limitation to TFP according to the manual, which makes cost calculation outside of research difficult.

\section{Widening Scope (Disorders and Age Groups)}

TFP started to provide an adapted and manualized psychoanalytic treatment for BPD, and there are further adaptions for the treatment of adolescents with Borderline features (TFP-Adolescence, Normandin, Ensink, Yeomans, \& Kernberg, 2014; Adolescent Identity Treatment, Foelsch et al., 2014) and other personality disorders such as Narcissistic Personality Disorder. Furthermore, the treatment approach was adapted for implementation in an acute psychiatric setting (Zerbo et al., 2013).

\section{A Process-Focused Psychodynamic Treatment - Mentalization-Based Treatment}

MBT is a manualized treatment protocol developed by Anthony Bateman and Peter Fonagy (Bateman \& Fonagy, 2016). The treatment is based on validating the emotional experience of patients within a significant therapeutic relationship and promotes several tech- 
niques that directly aim to stabilize or enhance mentalizing (Bateman \& Fonagy, 2016). Mentalization is the imaginative ability to interpret human behavior in terms of mental states (Fonagy, Gergely, Jurist, \& Target, 2002). Empirical research has shown that although social cognition is not necessarily impaired in BPD the construct of understanding others in emotionally intense relationships is highly impaired in BPD, which may underlie the core problems of these patients (Fonagy, Luyten, \& Strathearn, 2011). By promoting mentalizing, MBT addresses the interpersonal sensitivity in BPD.

\section{Clinical Concept}

Effective mentalizing is characterized by a genuine curiosity about mental states' underlying behavior, a flexibility in interpreting self and others as well as the knowledge that mentalizing is inaccurate most of the time and needs communication with others to clarify intentions more precisely. Furthermore, healthy mentalizing enables an individual to actively shift between different poles of mentalizing, e.g. self vs. others, integration of cognition and affect or implicit vs. explicit mentalizing. Patients with BPD are often overwhelmed by their emotions, make over-quick assumptions and focus on thinking about others with fears of abandonment and rejection. In MBT, the prototypical problems for working with patients with BPD are regarded as a sign of vulnerability in mentalizing that goes along with a high interpersonal sensitivity. An attachment threat leads to a breakdown in mentalizing, which leads to a failure of affect regulation and impulsive behavior. The vulnerability in mentalizing has been conceptualized as three different forms of inadequate mentalizing: teleological mode, psychic equivalence and pseudo-mentalizing. Teleological thinking overgeneralizes behavior as proof for internal states, whereas psychic equivalence generalizes from internal experience to the external reality. Pseudomentalizing creates mental theories without a connection between internal and external experience.

\section{Change Theory}

The proposed mechanism of change in MBT is to stabilize mentalizing in certain focus areas to create a psychic buffer between affect and behavior to foster affect regulation, reduce impulsivity and promote functional supportive relationships. This is reached by employing "contrary moves" to create more flexibility in using the different poles of mentalizing. If the patient is stuck in thinking about the self, the therapist will try to shift him or her towards thinking about others. If the patient is too certain about quickly made assumptions, the therapist will try to slow down and question the first assumption, etc. By sharing or disclosing the therapist's interpersonal experience with the patient from the beginning and throughout the process, the patient can find him/herself in the mind of the therapist and reflect on how the therapist is represented in the mind of the patient. Using constant empathic validation of the patient's affects and working slowly on cur- 
rent experiences with the therapist and other important others; the patient develops epistemic trust and is able to generalize helpful mentalizing experiences with the therapist to other relationships outside of therapy. Furthermore, by sharing a written case formulation, the patient learns about the therapist's idea of the patient's mentalizing failures and help him/her to establish more agency and responsibility for his/her behavior with regard to core symptoms e.g. self-harm, drug abuse.

\section{Setting}

MBT was initially developed as an inpatient treatment with a duration of 18 months and evolved to an intensive outpatient program that is now commonly limited to 12 months. MBT sets off with a diagnostic phase. In addition to standard diagnostic assessment, the clinician is assessing mentalizing problems and interpersonal triggers that are associated with the core problem behavior. This is written down in a case formulation that summarizes the clinician's current understanding of the patient's vulnerabilities and mentalizing problems all set in the context of current relationships and behavior. The case formulation is shared with the patient, serves as a focus for treatment, and is revised approx. every three months. In addition to the case formulation, a crisis plan is developed together with the patient entailing information which the patient finds helpful or hindering during breakdowns for him/herself, professionals and significant others. After the diagnostic phase the patient participates in a psycho-education group that teaches core elements of the treatment including an understanding of the BPD diagnosis. After 12 sessions the group changes its format to a MBT-group therapy focusing on elaborating perspectives from each group member. Parallel to the weekly group sessions patients have one weekly individual session.

\section{Stance}

Several aspects are essential for the MBT stance: being curious and enthusiastic for mental states, being authentic, empathic and validating as well as most importantly, taking a not-knowing stance. The latter is based on the modesty that no one can read minds and creates a less hierarchical relationship between therapist and patients. The therapist is not the expert for the patient's mind but rather takes an inquisitive stance to explore together with the patient what kind of thinking is helpful or unhelpful to have good relationships with others. Another focus is related to misunderstanding each other. Misunderstanding is considered as an opportunity to learn about perceptions, interpretations and experience. The therapist actively structures the session by focusing on topics related to the case formulation, management of arousal and monitoring the level of mentalizing. 


\section{Key Interventions}

Interventions start from the surface and work towards relational mentalizing of the therapeutic relationship if the current arousal and level of mentalizing allows. During times of high arousal it is recommended to intervene supportively by empathically validating the patient's subjective experience and addressing non-mentalizing by exploring affects, certainties, quick assumptions, and by challenging pseudo-mentalizing. The techniques are called "stop and stand" or "stop, rewind and explore" that slow down the processing of current experiences. Lower levels of arousal allow to start basic mentalizing around the focus of treatment such as triggers of strong affects and effect on behavior and others as well as linking different experiences to patterns of experience. Finally, exploring the current affect during the session (affect focus) and the relationship between therapist and patient are seen as crucial change mechanisms as this allows an understanding of interpersonal processes in the here-and-now. MBT deviates from classic psychoanalytic interpretations as this is regarded aversive for BPD patients. Thus, within the MBT framework it is recommended to contextualize affects and patterns of behavior in the hereand-now that should not be interpreted as a mere repetition of past relationships and experiences.

\section{Time and Costs}

Training comprises 5 days and four supervised cases with at least 24 sessions each and four sessions of supervision per case. Supervision and training add up to an overall cost of 1,600€ when following the requirements of the Anna-Freud-Centre in London but may vary country wise. Number, duration and frequency of MBT sessions based on one weekly group and one individual session ranges between 90 sessions in twelve months or 120 sessions in 18 months. MBT was originally developed as an inpatient treatment, which is more costly than the outpatient program. However, exact numbers have not been reported yet. A recent RCT in the Netherlands tested the efficacy between MBT outpatient and day-hospital, and reported a superiority in secondary outcomes for the more costly dayhospital treatment (Smits et al., 2019). Cost-effectiveness data comparing both settings is not available yet.

\section{Widening Scope (Disorders and Age Groups)}

Meanwhile, programs have been developed for adolescents with BPD (MBT-A), parents with BPD (MBT-Lighthouse), Conduct Disorder, Antisocial Personality Disorder, Eating Disorders, families (MBT-F), children (MBT-C) and hard to reach clients (AMBIT) (for an overview: Bateman \& Fonagy, 2019). 


\section{Efficacy of PD Treatments for BPD}

\section{Efficacy of TFP}

Three RCTs have demonstrated the efficacy of TFP. The first efficacy trial was conducted by Giesen-Bloo et al. (2006), with outpatients $(n=88)$ comparing TFP with Schema-Focused Therapy (SFT) with 2 weekly sessions over a duration of 3 years. Using an intention-to-treat approach, statistically and clinically significant improvements were found for both treatments on all measures after 1-, 2-, and 3-year treatment periods. However, SFT patients had a lower risk for drop-out $(\mathrm{RR}=0.52)$ and after 3 years of treatment, survival analyses demonstrated that significantly more SFT patients recovered or showed reliable clinical improvement. Robust analysis of covariance (ANCOVA) showed that they also improved more in general psychopathologic dysfunction and showed greater increases in quality of life. Arntz, Stupar-Rutenfrans, Bloo, van Dyck, and Spinhoven (2015) reanalyzed the Giessen-Bloo study and identified the following predictors for drop-out and reduced recovery: initial burden of dissociation, hostility and childhood physical abuse, whereby in-session dissociation (observer-report) was identified as a mediator. Another outpatient RCT with $n=90$ patients was conducted by Clarkin, Levy, Lenzenweger, and Kernberg (2007), who compared TFP (two individual weekly sessions) with DBT (weekly individual + group plus telephone consultation) and Dynamic Supportive Treatment (DST) (one individual weekly session) over a duration of 12 months. They found significant improvement for all three treatments on a number of outcomes: depression, anxiety, global functioning and social adjustment. No differences were found between the three different treatments; only TFP had a two times lower risk of drop-out (compare also Oud, Arntz, Hermens, Verhoef, \& Kendall, 2018, for a summary). Thereby individual slopes differed with regard to within-patient effects. Individual growth curve analysis showed that DBT and TFP had significant change rates compared to DST on suicidality, whereas TFP and DST had significant change rates compared to DBT on anger and impulsivity. Furthermore, only TFP showed significant change rates in aggression (direct and verbal assault) and irritability. Doering et al. (2010) investigated the efficacy of a TFP treatment compared to community treatment by experts (CTBE) over one year in $n=104$ female patients with BPD. In this trial, TFP showed superiority to CTBE with regard to reduced drop-out (38.5\% v. 67.3\%), suicide attempts, borderline symptomatology, increased psychosocial functioning, personality organization and psychiatric inpatient admissions. No differences between the two treatment conditions were observed for depression, anxiety and general psychopathology. However, self-harming behavior did not change in either group. In a further analysis of the same data by Fischer-Kern et al. (2015) significant improvements in reflective functioning was also found for the TFP vs. the TAU group with a medium between-group effect size $(d=0.45)$. 


\section{Efficacy of MBT}

Four RCTs have investigated the efficacy of MBT in comparison to psychiatric services (Bateman \& Fonagy, 1999), structured clinical services including supportive psychotherapy (Bateman \& Fonagy, 2009; Jørgensen et al., 2013) and in adolescents with non-suicidal self-injury (NSSI), who mainly fulfilled criteria for BPD (Rossouw \& Fonagy, 2012). MBT proved to be superior to TAU/ clinical management in NSSI, suicide attempts, psychiatric symptoms, and hospitalization (Bateman \& Fonagy, 1999, 2009; Rossouw \& Fonagy, 2012) as well as core BPD symptoms (Bales et al., 2012; Rossouw \& Fonagy, 2012). One independent RCT confirmed positive effects for MBT in comparison to supportive therapy for general functioning, suggesting that MBT may address core problems in BPD beyond NSSI and suicidality (Jørgensen et al., 2013). MBT is the only treatment for which superiority to clinical management was demonstrated in all primary outcome variables as well as achieving significantly higher levels of employment or academic/occupation training eight years after admission (Bateman \& Fonagy, 2008). Findings also demonstrate that MBT shows superiority over TAU for interpersonal problems and general functioning (Stoffers et al., 2012). In sum, MBT has demonstrated reliable improvements for psychiatric symptoms. A mediator analysis in an adolescent trial demonstrated that two changing variables were partially explaining differences in outcome between control and intervention group. These variables were changes in mentalizing and attachment avoidance, which were specific to the MBT effects (Rossouw \& Fonagy, 2012). In a recent naturalistic study with a sample of 175 patients with BPD treated in an inpatient setting, changes of mentalizing operationalized with the Reflective Functioning Questionnaire Uncertainty Scale (RFQ) were significantly associated with changes in outcome $(r=.89)$ (De Meulemeester, Vansteelandt, Luyten, \& Lowyck, 2018). This can be regarded as first evidence for a proposed specific change mechanism, i.e. changes in mentalizing mediate symptom improvement in BPD.

\section{Reviews and Meta-Analyses}

Seven systematic reviews on the general efficacy of psychological therapies for BPD (Brazier et al., 2006; Cristea et al., 2017; Juanmartí \& Lizeretti, 2017; Leichsenring et al., 2011; Oud et al., 2018; Stoffers et al., 2012) and therapy retention have been conducted, respectively (Barnicot, Katsakou, Marougka, \& Priebe, 2011). The Cochrane review (Stoffers et al., 2012) lists several approaches as 'probably effective' in treating BPD. Among those treatment approaches, MBT is the most frequently investigated after DBT. The authors recommend to conduct future trials with more than one psychological treatment and to include quality of life and preference measures across different programs.

In a recent meta-analysis investigating RCTs on psychotherapy efficacy in reducing suicidal attempts and NSSI (Calati \& Courtet, 2016), efficacy was established only for MBT compared to DBT, CBT, Cognitive Therapy and Interpersonal Psychotherapy. How- 
ever, results were based on the inclusion of only two MBT RCTs. In an updated metaanalysis, Cristea et al. (2017) with $k=33$ studies ( $n=2,256$ patients) conclude that only DBT and psychodynamic approaches were more effective than control interventions, however risk of bias was a significant moderator and publication bias was persistent particularly at follow-up. McLaughlin, Barkowski, Burlingame, Strauss, and Rosendahl (2019) investigate in $\mathrm{k}=24$ RCTs with over $\mathrm{n}=1,500$ patients the efficacy of group psychotherapy for BPD and find that group psychotherapy has a large effect on the reduction of BPD symptoms and a moderate effect on suicidality/ parasuicidal symptoms. While the largest numbers of studies available have investigated DBT, theoretical orientation of treatment was not a significant moderator for BPD symptoms in this meta-analysis. McLaughlin et al. (2019) conclude that dismantling studies, investigating the effect of various treatment components are promising. Leichsenring et al. (2011) and the Cochrane review (Stoffers et al., 2012) criticize the low study quality across BPD trials due to researcher allegiance, attention bias and small samples. They conclude that there is a strong need for confirmatory trials with high study quality and sufficient sample sizes.

Oud et al. (2018) summarize in their review and meta-analysis RCTs on DBT, MBT, TFP and ST to compare specialized therapies for BPDs with non-specialized treatments. When pooling comparison data from specialized treatments vs. community treatment by experts, they demonstrate that specialized psychodynamic treatments like MBT or TFP are superior to non-specialized psychodynamic treatment with regard to overall BPD severity and drop-out. With regard to self-injury TFP showed no superiority and with regard to suicidality both DBT and TFP were no better than community expert therapists. However, these results have to be interpreted cautiously as they are based on three trials only.

\section{Similarities and Differences Between MBT and TFP}

Both treatment approaches are regarded as evidence-based and are gathering further proof in ongoing trials. So far, mechanisms of change have not been empirically established; however, this is, unfortunately, currently the case for all psychotherapies. As no study has directly compared TFP and MBT so far, it is unclear if one is more effective than the other or more suited for BPD and respective subgroups. Thus, a differential indication for the treatment of patients with BPD cannot be made based on empirical findings. There is no evidence that allows to choose which psychotherapy may be the most appropriate for which patient profile (Fonagy, Luyten, \& Bateman, 2017). Aside from $\mathrm{BPD}$, the widening scope of treating other personality disorders reveals recommendations for treating patients with internalizing personality disorders with TFP (e.g. Narcissistic PD) (Kernberg, 2016) and patients with externalizing personality disorders with MBT (e.g. ASPD) (Bateman, O'Connell, Lorenzini, Gardner, \& Fonagy, 2016). 
TFP and MBT are based on different clinical and theories of change. TFP is more stringently rooted in classic psychoanalytic theory and jargon, while MBT created a new conceptual framework by bridging several theoretical underpinnings from psychoanalysis, attachment theory and general developmental psychology. Differences can also be found with regard to the setting: while TFP deviates from classic psychoanalysis only by not using the couch and reducing the weekly frequency to one to two hours, MBT has integrated psycho-education and group therapy which may create less pressure or intensity. However, the dyadic therapeutic work itself appears quite similar even though both approaches use different terminology for their interventions. Especially, clarification and confrontation in TFP are very close to exploration, clarification and challenge in MBT. Furthermore, establishing a contract and crisis plan at the beginning of therapy, working in the here-and-now, using the therapeutic relationship as a training ground and monitoring the therapist's counter-reaction is required in both therapies.

Major differences can be found in the general therapeutic stance that each approach is advocating. A TFP therapist is asked to remain in technical neutrality (not taking a stance towards or against any content discussed). On the contrary, the MBT therapist is asked to be enthusiastic and praising for mentalizing as well as disclosing his/her emotions if this is regarded as helpful to create a mentalizing process. While TFP is deploying a content-focused approach taking an interpretative expressive therapeutic stance, MBT focuses on the process of thinking about mental states based on a supportive therapeutic stance. Yet again, there is also a considerable overlap: Interestingly, TFP also increases mentalizing (Fischer-Kern et al., 2015; Levy et al., 2006), which may be evidence that the core therapeutic work of clarification and confrontation and maybe also interpretation creates robust mentalizing. As mechanism of change studies in MBT reveal that increasing reflective thinking is indeed mediating symptomatic improvement in BPD, this could also be interpreted as a common change factor across treatments in BPD (Goodman, 2013). Hence, it would be worth investigating these specific differences and similarities in process research as well as within non-inferiority trials to test the efficacy.

Dissemination of MBT and TFP is a major challenge as many psychodynamic therapists are skeptical towards disorder-specific treatment and variations from a highly individualized and transdiagnostic approach that is typical for psychodynamic therapies (Gonzalez-Torres, 2018). Furthermore, accredited supervisors and trainers are still scarce for both TFP and MBT, and this significantly hinders the international dissemination of training programs. However, considering the substantial burden of these patients, their need for adequate treatment and the substantial evidence supporting the efficacy of these treatments, advancing dissemination of treatment and empirical knowledge seems to be a worthwhile future investigation. 
Funding: The authors received no financial support for the research, authorship, and/or publication of this article.

Competing Interests: The authors declare no conflicts of interest.

Acknowledgments: The authors have no support to report.

\section{References}

American Psychiatric Association. (2013). Diagnostic and statistical manual of mental disorders (5th ed.). Washington, DC, USA: Author.

Arntz, A., Stupar-Rutenfrans, S., Bloo, J., van Dyck, R., \& Spinhoven, P. (2015). Prediction of treatment discontinuation and recovery from Borderline Personality Disorder: Results from an RCT comparing schema therapy and transference focused psychotherapy. Behaviour Research and Therapy, 74, 60-71. https://doi.org/10.1016/j.brat.2015.09.002

Bales, D., van Beek, N., Smits, M., Willemsen, S., Busschbach, J. J. V., Verheul, R., \& Andrea, H. (2012). Treatment outcome of 18-month, day hospital mentalization-based treatment (MBT) in patients with severe borderline personality disorder in the Netherlands. Fournal of Personality Disorders, 26(4), 568-582. https://doi.org/10.1521/pedi.2012.26.4.568

Barnicot, K., Katsakou, C., Marougka, S., \& Priebe, S. (2011). Treatment completion in psychotherapy for borderline personality disorder: A systematic review and meta-analysis. Acta Psychiatrica Scandinavica, 123(5), 327-338. https://doi.org/10.1111/j.1600-0447.2010.01652.x

Bateman, A., \& Fonagy, P. (1999). Effectiveness of partial hospitalization in the treatment of borderline personality disorder: A randomized controlled trial. The American fournal of Psychiatry, 156(10), 1563-1569. https://doi.org/10.1176/ajp.156.10.1563

Bateman, A., \& Fonagy, P. (2008). 8-year follow-up of patients treated for borderline personality disorder: Mentalization-based treatment versus treatment as usual. The American fournal of Psychiatry, 165(5), 631-638. https://doi.org/10.1176/appi.ajp.2007.07040636

Bateman, A., \& Fonagy, P. (2009). Randomized controlled trial of outpatient mentalization-based treatment versus structured clinical management for borderline personality disorder. The American fournal of Psychiatry, 166(12), 1355-1364. https://doi.org/10.1176/appi.ajp.2009.09040539

Bateman, A., \& Fonagy, P. (2016). Mentalization-based treatment for personality disorders: A practical guide. Oxford, United Kingdom: Oxford University Press.

Bateman, A., \& Fonagy, P. (Eds.). (2019). Handbook of mentalizing in mental health practice (2nd ed.). Washington, DC, USA: American Psychiatric Association Publishing.

Bateman, A., O'Connell, J., Lorenzini, N., Gardner, T., \& Fonagy, P. (2016). A randomised controlled trial of mentalization-based treatment versus structured clinical management for patients with comorbid borderline personality disorder and antisocial personality disorder. BMC Psychiatry, 16, Article 304. https://doi.org/10.1186/s12888-016-1000-9 
Brazier, J. E., Tumur, I., Holmes, M., Ferriter, M., Parry, G., Dent-Brown, K., \& Paisley, S. (2006). Psychological therapies including dialectical behaviour therapy for borderline personality disorder: A systematic review and preliminary economic evaluation (NIHR Health Technology Assessment programme: Executive Summaries). Southampton, United Kingdom.

Calati, R., \& Courtet, P. (2016). Is psychotherapy effective for reducing suicide attempt and nonsuicidal self-injury rates? Meta-analysis and meta-regression of literature data. fournal of Psychiatric Research, 79, 8-20. https://doi.org/10.1016/j.jpsychires.2016.04.003

Clarkin, J. F., Cain, N. M., \& Lenzenweger, M. F. (2018). Advances in transference-focused psychotherapy derived from the study of borderline personality disorder: Clinical insights with a focus on mechanism. Current Opinion in Psychology, 21, 80-85.

https://doi.org/10.1016/j.copsyc.2017.09.008

Clarkin, J. F., Levy, K. N., Lenzenweger, M. F., \& Kernberg, O. F. (2007). Evaluating three treatments for borderline personality disorder: A multiwave study. The American fournal of Psychiatry, 164(6), 922-928. https://doi.org/10.1176/ajp.2007.164.6.922

Cristea, I. A., Gentili, C., Cotet, C. D., Palomba, D., Barbui, C., \& Cuijpers, P. (2017). Efficacy of psychotherapies for borderline personality disorder: A systematic review and meta-analysis. FAMA Psychiatry, 74(4), 319-328. https://doi.org/10.1001/jamapsychiatry.2016.4287

De Meulemeester, C., Vansteelandt, K., Luyten, P., \& Lowyck, B. (2018). Mentalizing as a mechanism of change in the treatment of patients with borderline personality disorder: A parallel process growth modeling approach. Personality Disorders, 9(1), 22-29. https://doi.org/10.1037/per0000256

Doering, S., Hörz, S., Rentrop, M., Fischer-Kern, M., Schuster, P., Benecke, C., . . Buchheim, P. (2010). Transference-focused psychotherapy v. treatment by community psychotherapists for borderline personality disorder: Randomised controlled trial. The British fournal of Psychiatry, 196(5), 389-395. https://doi.org/10.1192/bjp.bp.109.070177

Fischer-Kern, M., Doering, S., Taubner, S., Hörz, S., Zimmermann, J., Rentrop, M., . . Buchheim, A. (2015). Transference-focused psychotherapy for borderline personality disorder: Change in reflective function. The British fournal of Psychiatry, 207(2), 173-174.

https://doi.org/10.1192/bjp.bp.113.143842

Foelsch, P., Schlüter-Müller, S., Odom, A., Arena, H., Borzutzky H. A., \& Schmeck, K. (2014). Adolescent identity treatment. Basel, Switzerland: Springer International Publishing.

Fonagy, P., Gergely, G., Jurist, E. J., \& Target, M. (2002). Affect regulation, mentalization and the development of the self. London, United Kingdom: Karnac Books.

Fonagy, P., Luyten, P., \& Bateman, A. (2017). Treating borderline personality disorder with psychotherapy: Where do we go from here? JAMA Psychiatry, 74(4), 316-317. https://doi.org/10.1001/jamapsychiatry.2016.4302

Fonagy, P., Luyten, P., \& Strathearn, L. (2011). Borderline personality disorder, mentalization, and the neurobiology of attachment. Infant Mental Health fournal, 32(1), 47-69.

https://doi.org/10.1002/imhj.20283 
Giesen-Bloo, J., van Dyck, R., Spinhoven, P., van Tilburg, W., Dirksen, C., van Asselt, T., . . Arntz, A. (2006). Outpatient psychotherapy for borderline personality disorder: Randomized trial of schema-focused therapy vs transference-focused psychotherapy. Archives of General Psychiatry, 63(6), 649-658. https://doi.org/10.1001/archpsyc.63.6.649

Gonzalez-Torres, M. A. (2018). Psychodynamic psychotherapies for borderline personality disorders. Current developments and challenges ahead. BfPsych International, 15(1), 12-14. https://doi.org/10.1192/bji.2017.7

Goodman, G. (2013). Is mentalization a common process factor in transference-focused psychotherapy and dialectical behavior therapy sessions? Journal of Psychotherapy Integration, 23(2), 179-192. https://doi.org/10.1037/a0032354

Grant, B. F., Chou, S. P., Goldstein, R. B., Huang, B., Stinson, F. S., Saha, T. D., . . Ruan, W. J. (2008). Prevalence, correlates, disability, and comorbidity of DSM-IV borderline personality disorder: Results from the Wave 2 National Epidemiologic Survey on Alcohol and Related Conditions. The fournal of Clinical Psychiatry, 69(4), 533-545. https://doi.org/10.4088/JCP.v69n0404

Gunderson, J. G., Weinberg, I., \& Choi-Kain, L. (2013). Borderline personality disorder. Focus, 11(2), 129-145. https://doi.org/10.1176/appi.focus.11.2.129

Jobst, A., Hörz, S., Birkhofer, A., Martius, P., \& Rentrop, M. (2010). Psychotherapists' attitudes towards the treatment of patients with borderline personality disorder. Psychotherapie, Psychosomatik, Medizinische Psychologie, 60(3/04), 126-131.

https://doi.org/10.1055/s-0029-1220764

Jørgensen, C. R., Freund, C., Bøye, R., Jordet, H., Andersen, D., \& Kjølbye, M. (2013). Outcome of mentalization-based and supportive psychotherapy in patients with borderline personality disorder: A randomized trial. Acta Psychiatrica Scandinavica, 127(4), 305-317. https://doi.org/10.1111/j.1600-0447.2012.01923.x

Juanmartí, F. B., \& Lizeretti, N. P. (2017). Eficacia de la psicoterapia para el tratamiento del Trastorno Límite de la Personalidad: Una revisión. Papeles Del Psicólogo - Psychologist Papers, 37(1), 148-156. https://doi.org/10.23923/pap.psicol2017.2832

Kernberg, O. (1967). Borderline personality organization. Journal of the American Psychoanalytic Association, 15(3), 641-685. https://doi.org/10.1177/000306516701500309

Kernberg, O. F. (2016). New developments in transference focused psychotherapy. The International Fournal of Psycho-Analysis, 97(2), 385-407. https://doi.org/10.1111/1745-8315.12289

Leichsenring, F., Leibing, E., Kruse, J., New, A. S., \& Leweke, F. (2011). Borderline personality disorder. The Lancet, 377(9759), 74-84. https://doi.org/10.1016/S0140-6736(10)61422-5

Lemma, A., Target, M., \& Fonagy, P. (2011). The development of a brief psychodynamic intervention (dynamic interpersonal therapy) and its application to depression: A pilot study. Psychiatry, 74(1), 41-48. https://doi.org/10.1521/psyc.2011.74.1.41

Levy, K. N., Meehan, K. B., Kelly, K. M., Reynoso, J. S., Weber, M., Clarkin, J. F., . . Kernberg, O. F. (2006). Change in attachment patterns and reflective function in a randomized control trial of transference-focused psychotherapy for borderline personality disorder. fournal of Consulting and Clinical Psychology, 74, 1027-1040. https://doi.org/10.1037/0022-006X.74.6.1027 
Luyten, P., Blatt, S. J., \& Fonagy, P. (2013). Impairments in self structures in depression and suicide in psychodynamic and cognitive behavioral approaches: Implications for clinical practice and research. International fournal of Cognitive Therapy, 6(3), 265-279. https://doi.org/10.1521/ijct.2013.6.3.265

McLaughlin, S. P. B., Barkowski, S., Burlingame, G. M., Strauss, B., \& Rosendahl, J. (2019). Group psychotherapy for borderline personality disorder: A meta-analysis of randomized-controlled trials. Psychotherapy, 56(2), 260-273. https://doi.org/10.1037/pst0000211

Milrod, B., Leon, A. C., Busch, F., Rudden, M., Schwalberg, M., Clarkin, J., . . Shear, M. K. (2007). A randomized controlled clinical trial of psychoanalytic psychotherapy for panic disorder. The American fournal of Psychiatry, 164(2), 265-272. https://doi.org/10.1176/ajp.2007.164.2.265

Normandin, L., Ensink, K., Yeomans, F. E., \& Kernberg, O. F. (2014). Transference-focused psychotherapy for personality disorders in adolescence. In C. Sharp \& J. L. Tackett (Eds.), Handbook of borderline personality disorder in children and adolescents (pp. 333-359). New York, NY, USA: Springer.

Oldham, J. M. (2006). Borderline personality disorder and suicidality. The American fournal of Psychiatry, 163(1), 20-26. https://doi.org/10.1176/appi.ajp.163.1.20

Olesen, J., Gustavsson, A., Svensson, M., Wittchen, H.-U., \& Jönsson, B. (2012). The economic cost of brain disorders in Europe. European fournal of Neurology, 19(1), 155-162.

https://doi.org/10.1111/j.1468-1331.2011.03590.x

OPD Taskforce. (Eds.). (2008). Operationalized Psychodynamic Diagnosis OPD-2: Manual of diagnosis and treatment planning. Cambridge, MA, USA: Hogrefe \& Huber.

Oud, M., Arntz, A., Hermens, M. L., Verhoef, R., \& Kendall, T. (2018). Specialized psychotherapies for adults with borderline personality disorder: A systematic review and meta-analysis. The Australian and New Zealand fournal of Psychiatry, 52(10), 949-961. https://doi.org/10.1177/0004867418791257

Rossouw, T. I., \& Fonagy, P. (2012). Mentalization-based treatment for self-harm in adolescents: A randomized controlled trial. Journal of the American Academy of Child and Adolescent Psychiatry, 51(12), 1304-1313.e3. https://doi.org/10.1016/j.jaac.2012.09.018

Salvador-Carulla, L., Bendeck, M., Ferrer, M., Andión, O., Aragonès, E., \& Casas, M. (2014). Cost of borderline personality disorder in Catalonia (Spain). European Psychiatry, 29(8), 490-497. https://doi.org/10.1016/j.eurpsy.2014.07.001

Shedler, J. (2010). The efficacy of psychodynamic psychotherapy. American Psychologist, 65(2), 98-109. https://doi.org/10.1037/a0018378

Smits, M. L., Feenstra, D. J., Eeren, H. V., Bales, D. L., Laurenssen, E. M. P., Blankers, M., . . Luyten, P. (2019). Day hospital versus intensive out-patient mentalisation-based treatment for borderline personality disorder: Multicentre randomised clinical trial. The British fournal of Psychiatry. Advance online publication. https://doi.org/10.1192/bjp.2019.9

Stoffers, J. M., Völlm, B. A., Rücker, G., Timmer, A., Huband, N., \& Lieb, K. (2012). Psychological therapies for people with borderline personality disorder. The Cochrane Database of Systematic Reviews, 8, Article CD005652. https://doi.org/10.1002/14651858.CD005652.pub2 
Torgersen, S. (2005). Epidemiology. In J. M. Oldham, A. E. Skodol, \& D. S. Bender (Eds.), The American Psychiatric Publishing textbook of personality disorders (pp. 129-141). Washington, DC, USA: American Psychiatric Publishing.

Trull, T. J., Jahng, S., Tomko, R. L., Wood, P. K., \& Sher, K. J. (2010). Revised NESARC personality disorder diagnoses: Gender, prevalence, and comorbidity with substance dependence disorders. Fournal of Personality Disorders, 24(4), 412-426. https://doi.org/10.1521/pedi.2010.24.4.412

van Asselt, A. D. I., Dirksen, C. D., Arntz, A., Giesen-Bloo, J. H., van Dyck, R., Spinhoven, P., . . Severens, J. L. (2008). Out-patient psychotherapy for borderline personality disorder: Costeffectiveness of schema-focused therapy v. transference-focused psychotherapy. The British fournal of Psychiatry, 192(6), 450-457. https://doi.org/10.1192/bjp.bp.106.033597

Victorian Government Department of Human Services. (2005). Victorian Burden of Disease Study. Melbourne, Australia: Author.

Volkert, J., Gablonski, T.-C., \& Rabung, S. (2018). Prevalence of personality disorders in the general adult population in Western countries: Systematic review and meta-analysis. The British Journal of Psychiatry, 213(6), 709-715. https://doi.org/10.1192/bjp.2018.202

Wagner, T., Roepke, S., Marschall, P., Stiglmayr, C., Renneberg, B., Gieb, D., . . Fydrich, T. (2013). Krankheitskosten der Borderline Persönlichkeitsstörung aus gesellschaftlicher Perspektive. Zeitschrift für Klinische Psychologie und Psychotherapie, 42(4), 242-255. https://doi.org/10.1026/1616-3443/a000227

Whitehorn, J. C., Braceland, F. J., Lippard, V. W., \& Malamud, W. (Eds.). (1953). The Psychiatrist: His training and development. Washington, DC, USA: American Psychiatric Association.

Yeomans, F. E., Clarkin, J. F., \& Kernberg, O. F. (2015). Transference-focused psychotherapy for borderline personality disorder: A clinical guide. Washington, DC, USA: American Psychiatric Publishing.

Yeomans, F. E., Levy, K. N., \& Caligor, E. (2013). Transference-focused psychotherapy. Psychotherapy, 50(3), 449-453. https://doi.org/10.1037/a0033417

Zerbo, E., Cohen, S., Bielska, W., \& Caligor, E. (2013). Transference-focused psychotherapy in the general psychiatry residency: A useful and applicable model for residents in acute clinical settings. Psychodynamic Psychiatry, 41(1), 163-181. https://doi.org/10.1521/pdps.2013.41.1.163 


\section{EACLIPT}

Clinical Psychology in Europe (CPE) is the official journal of the European Association of Clinical Psychology and Psychological Treatment (EACLIPT).

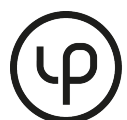

leibniz-psychology.org

PsychOpen GOLD is a publishing service by Leibniz Institute for Psychology Information (ZPID), Germany. 